\title{
The History of the Cluster Heat Map
}

\section{Leland WILKINSON and Michael FRIENDLY}

The cluster heat map is an ingenious display that simultaneously reveals row and column hierarchical cluster structure in a data matrix. It consists of a rectangular tiling, with each tile shaded on a color scale to represent the value of the corresponding element of the data matrix. The rows (columns) of the tiling are ordered such that similar rows (columns) are near each other. On the vertical and horizontal margins of the tiling are hierarchical cluster trees. This cluster heat map is a synthesis of several different graphic displays developed by statisticians over more than a century. We locate the earliest sources of this display in late 19th century publications, and trace a diverse 20th century statistical literature that provided a foundation for this most widely used of all bioinformatics displays.

KEY WORDS: Cluster analysis; Heatmap; Microarray; Visualization.

\section{INTRODUCTION}

The cluster heat map is a rectangular tiling of a data matrix with cluster trees appended to its margins. Within a relatively compact display area, it facilitates inspection of row, column, and joint cluster structure. Moderately large data matrices (several thousand rows/columns) can be displayed effectively on a high-resolution color monitor, and even larger matrices can be handled in print or in megapixel displays.

The cluster heat map is well known in the natural sciences and is one of the most widely used graphs in the biological sciences. As Weinstein (2008) notes,

For visualization, by far the most popular graphical representation has been the clustered heat map, which compacts large amounts of information into a small space to bring out coherent patterns in the data.... Since their debut over 10 years ago, clustered heat maps have appeared in well over 4000 biological or biomedical publications.

Weinstein describes the heat map as follows:

In the case of gene expression data, the color assigned to a point
in the heat map grid indicates how much of a particular RNA
or protein is expressed in a given sample. The gene expression

Leland Wilkinson is Executive Vice President, SYSTAT Inc., Chicago, IL 60622 (E-mail and Web: Leland.wilkinson@systat.com, http://www.cs.uic. edu/ wilkinson/index.html). Michael Friendly is Professor, Department of Psychology, York University, Toronto, Ontario M3J 1P3, Canada (E-mail and Web: friendly@yorku.ca, http://www.math.yorku.ca/SCS/friendly.html).

\begin{abstract}
level is generally indicated by red for high expression and either green or blue for low expression. Coherent patterns (patches) of color are generated by hierarchical clustering on both horizontal and vertical axes to bring like together with like. Cluster relationships are indicated by tree-like structures adjacent to the heat map, and the patches of color may indicate functional relationships among genes and samples.
\end{abstract}

Figure 1 shows a typical heat map as described by Weinstein. The most popular bioinformatics software for producing this graphic was documented by Eisen et al. (1998). That article, which describes a cluster heat map program, was the third most cited article in PNAS as of July 1, 2008 (PNAS 2008).

The "debut" to which Weinstein refers is possibly a debut in the biology literature, but it certainly is not a debut in the statistical literature. The components of this display have a long history in statistical graphics. The biological references give little indication of the background for the underlying ideas required to construct a heat map. In this article we trace the lineage of the heat map and show what elements were ultimately integrated in the display that biologists finally adopted.

\section{THE PAST}

To elucidate the history of this display, we present each of the components underlying the design of the cluster heat map. Some are quite old, some relatively recent.

\subsection{Shading Matrices}

The heart of the heat map is a color-shaded matrix display. Shaded matrix displays are well over a century old. Figure 2 shows an example from Loua (1873) that summarizes various social statistics across the arrondissements of Paris. Like the other graphics in the book, it was drawn and colored by hand.

Shading a table or matrix is a long-established method of highlighting entries, rows, or columns. Accountants, graphics designers, computer engineers, and others have used this method for years. The most common recent application involves the use of color to shade rows, columns, or cells of a spreadsheet.

\subsection{Permuting Matrices}

The cluster heat map does more than shade; it permutes the rows and columns of a matrix to reveal structure. Matrix permutation has a long history as well. Like the idea of shading, sorting a matrix or table to reveal structure is more than a century old. Figure 3 shows a sorted matrix of educational data from Brinton (1914). Figure 4 shows an example from Bertin 


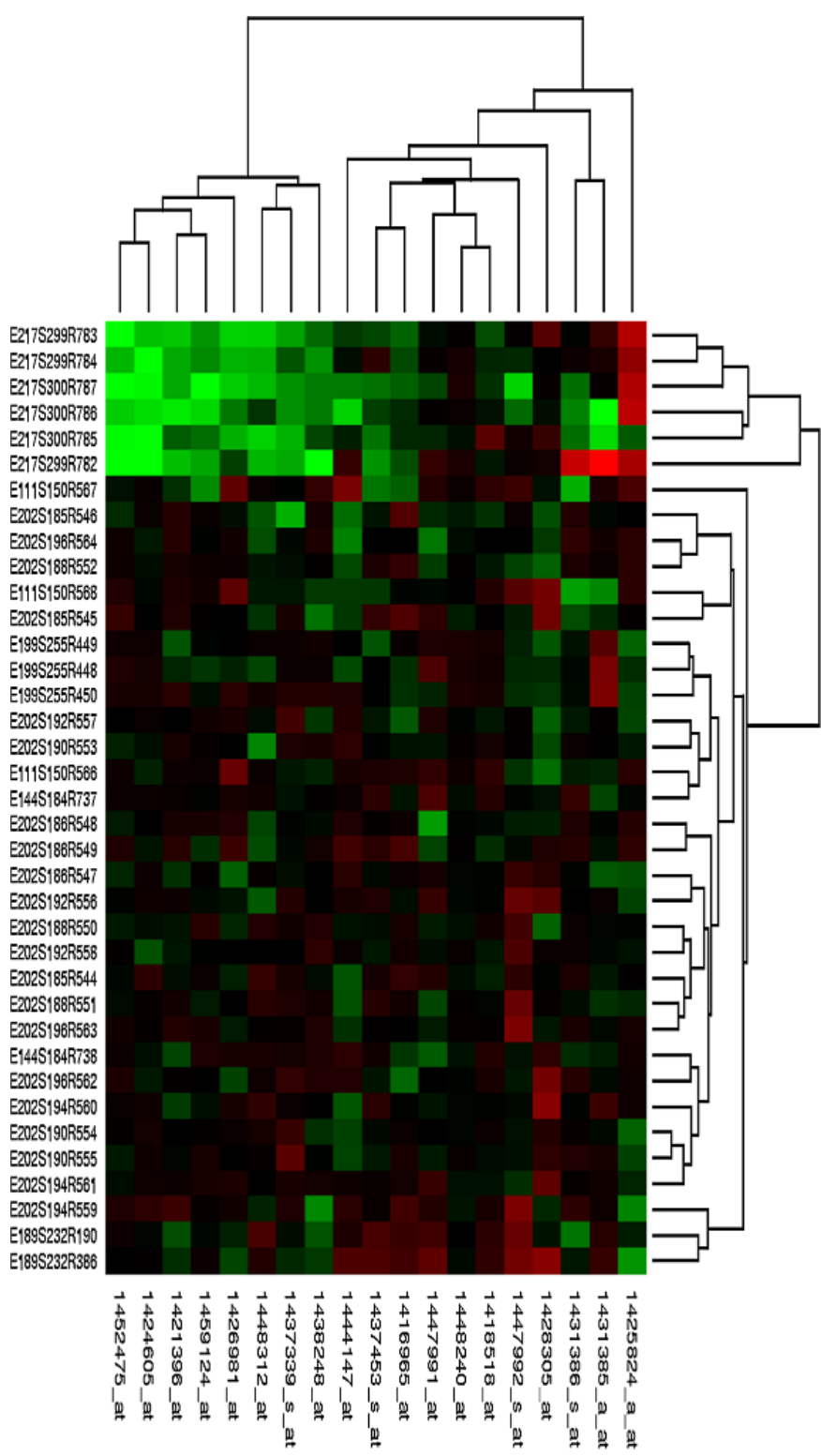

Figure 1. Cluster heat map from Andrade (2008), based on Eisen et al. (1998). The aspect ratio has been adjusted to make the pixels square. The rows (or columns) of a microarray heat map represent genes, and the columns (or rows) represent samples. Each cell is colorized based on the level of expression of that gene in that sample.

(1967). Jacques Bertin devoted a chapter to illustrating the usefulness of what he called the reorderable matrix. His examples were sorted by hand.

\subsubsection{Seriation}

It was an anthropologist who developed one of the first models for ordering a data matrix. Petrie (1899) sought to rearrange the rows and columns of a rectangular matrix of measurements on anthropological artifacts so that the largest values would be near the main diagonal. His immediate goal was to use attributes (columns) to serialize artifacts (rows) to recover a temporal ordering on the artifacts. His goal had implications well beyond his subject matter. Petrie identified the Toeplitz structure implicit in the ordering of a data matrix based on time (or some other dimension). His article generated a large literature over more than a century on a topic variously called seriation or matrix reordering (Robinson 1951; Kendall 1963; McCormick, Schweitzer, and White 1972; Hubert 1974, 1976; Lenstra 1974; Friendly 2002; Friendly and Kwan 2003; Climer and Zhang 2006).

Ten years after the publication of Petrie's article, Jan Czekanowski developed a seriation method and used a shaded diagram to represent block-diagonal data structures. Figure 5 shows a sorted matrix of educational data from Czekanowski (1909). Except for its lack of coloring and appended cluster trees, Czekanowski's display is similar to the output of contemporary computer matrix reordering programs (Liiv 2008).

\subsubsection{The Guttman Scalogram}

Fifty years after Petrie, Louis Guttman introduced a matrix permutation to reveal a different one-dimensional structure. The Guttman scalogram (Guttman 1950) is a direct method for fitting a deterministic model (a total order that Guttman called a "simplex") to a binary matrix. In Guttman's method, a rectangular binary matrix is permuted by hand (using paper or a tabulating machine) to approximate a unidimensional scale: below the quasi-diagonal are as many 1 's as possible and above the quasi-diagonal are as many 0's as possible. A matrix with this structure is said to be scalable, implying an ordering of the rows and columns.

The scalogram found wide application in subsequent decades, particularly in the social sciences. Figure 6 shows an example from Rondinelli (1980). Computer programs eventually automated this scaling (Nie, Bent, and Hull 1970; Wilkinson 1979). Others eventually developed interactive visual analytics programs to allow users to explore their own permutations (Siirtola and Mäkinen 2005). Statisticians developed stochastic generalizations of Guttman's model that allowed this permutation to be applied more widely (Goodman 1975; Andrich 1978).

\subsubsection{Hierarchical Clustering}

Not long after Guttman's scalogram became popular, cluster analysts took an interest in representing clusters by shading association (similarity/dissimilarity) matrices. Sneath (1957) was perhaps the earliest advocate for this graphic.

Ling (1973) introduced a computer program, called SHADE, for implementing Sneath's idea. Ling's program used overstrikes on a character printer to represent different degrees of shading. Gower and Digby (1981) implemented Ling's display on a dot matrix printer; Figure 7 shows an example.

\subsubsection{Two-Way Clustering}

Shortly after the publication of Ling's article, Hartigan (1974) introduced a block clustering program with direct display of a rectangular data matrix. The theory behind this program was discussed by Hartigan (1975). Motivated by Hartigan's work, Wilkinson (1984) implemented a two-way hierarchical clustering routine on a rectangular data matrix, using Ling's shading method for the display. 


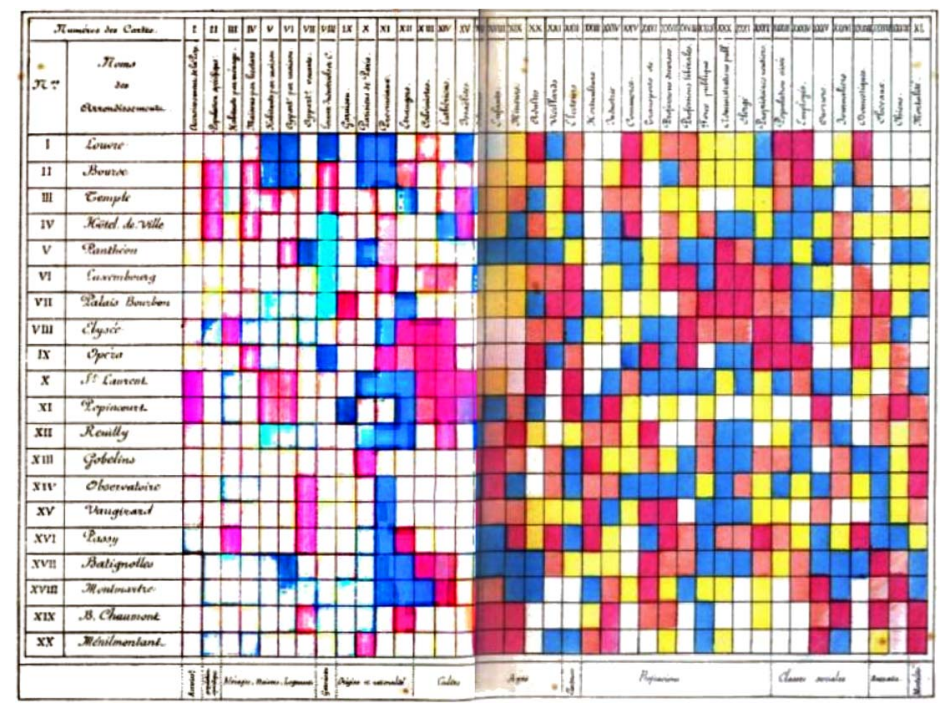

Figure 2. Shaded matrix display from Loua (1873), available online at http://books.google.com/books/. This was designed as a summary of 40 separate maps of Paris, showing the characteristics (e.g., national origin, professions, age, social classes) of 20 districts, using a color scale ranging from white (low) through yellow and blue to red (high).
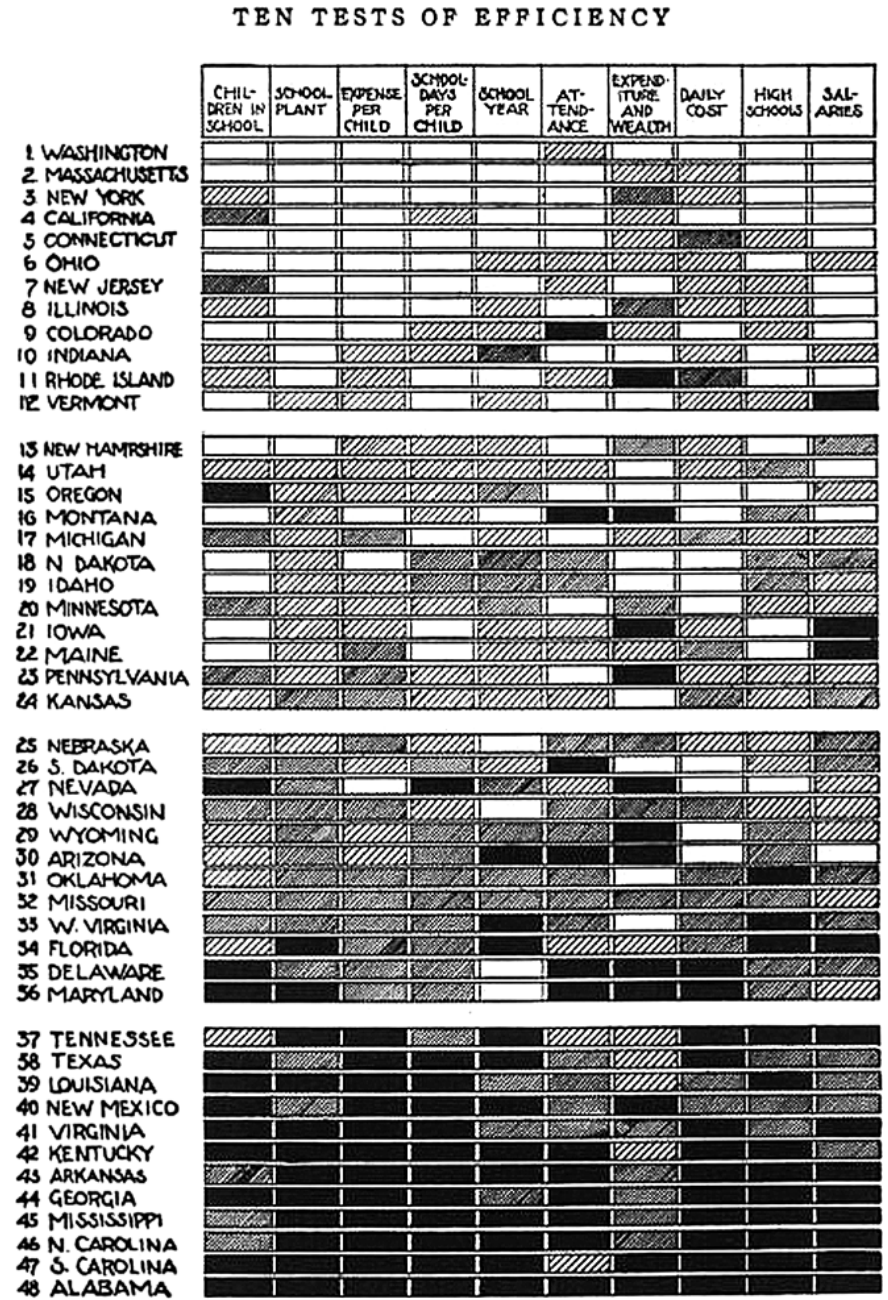

Figure 3. Sorted shaded display from Brinton (1914). The data are ranks of U.S. states on each of 10 educational features assessed in 1910. The matrix has been sorted by the row-marginal ranks.
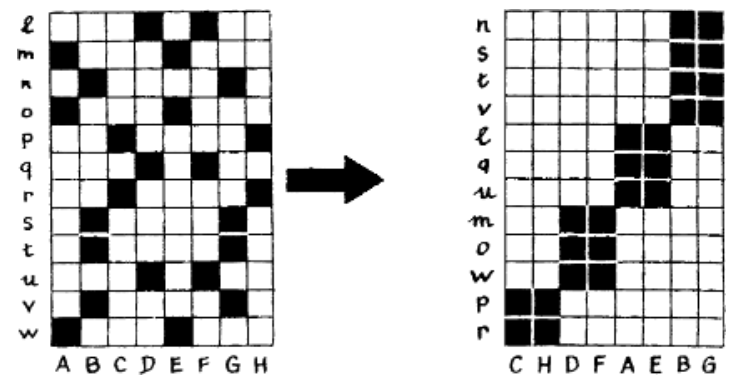

Figure 4. Permuted matrix display from Bertin (1967). This figure was devised to illustrate the possibility of sorting a matrix to reveal block-diagonal structure.

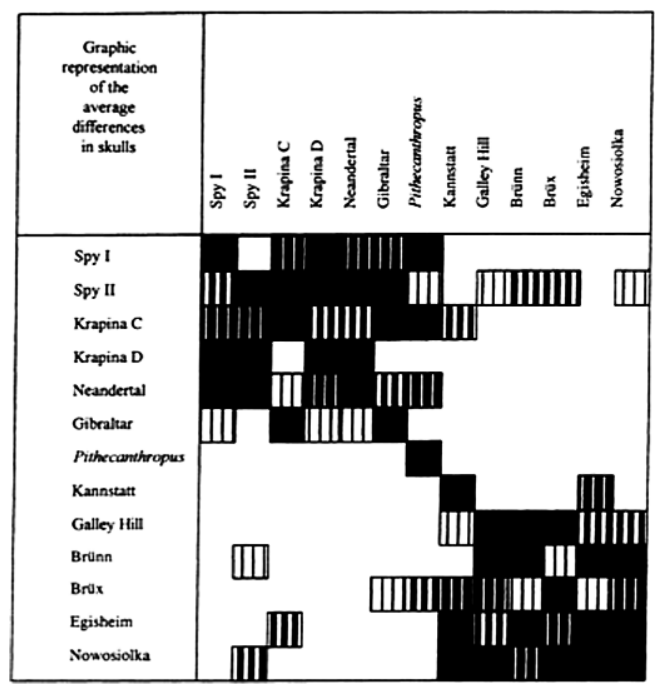

Figure 5. Sorted shaded display from Czekanowski (1909), reproduced in Hage and Harary (1995). 

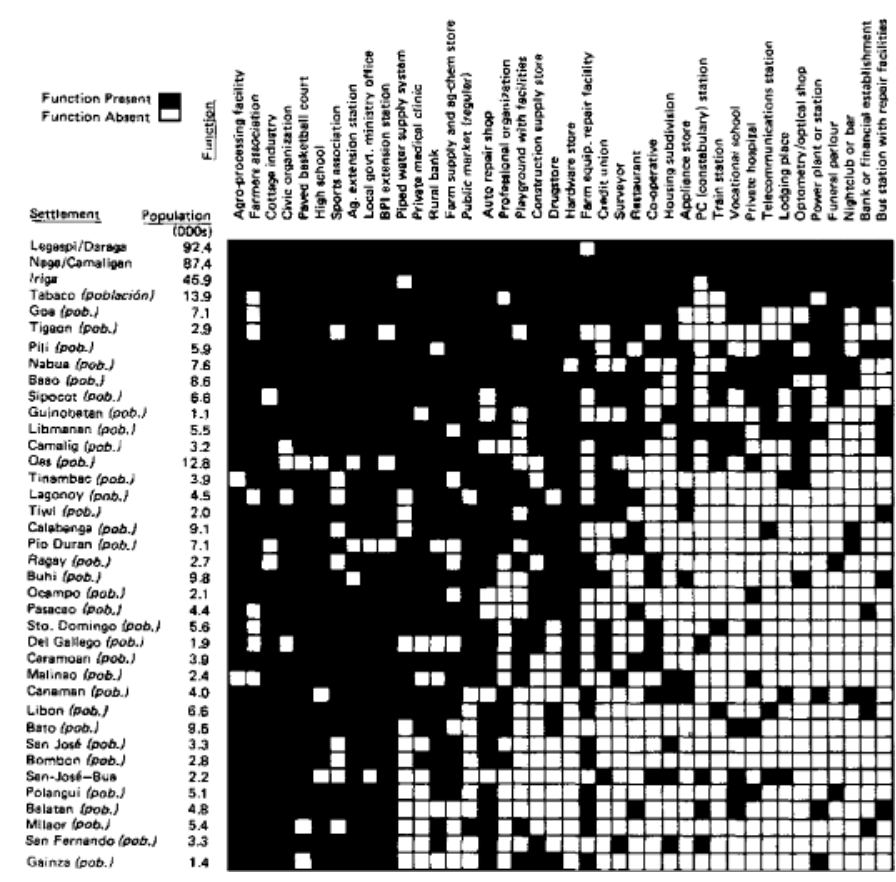

Figure 6. Scalogram display from Rondinelli (1980), based on Guttman (1950). This manually-sorted scalogram summarizes facilities statistics (e.g., high school, rural bank, auto repair shop, drugstore) for settlements in the Bicol River Basin, Phillipines.

\subsubsection{Seriating a Binary Tree}

For a binary tree with $\mathrm{n}$ leaves, there are $2^{\mathrm{n}-1}$ possible linear orderings of the leaves in a planar layout of the tree. Hierarchical clustering algorithms do not determine a particular

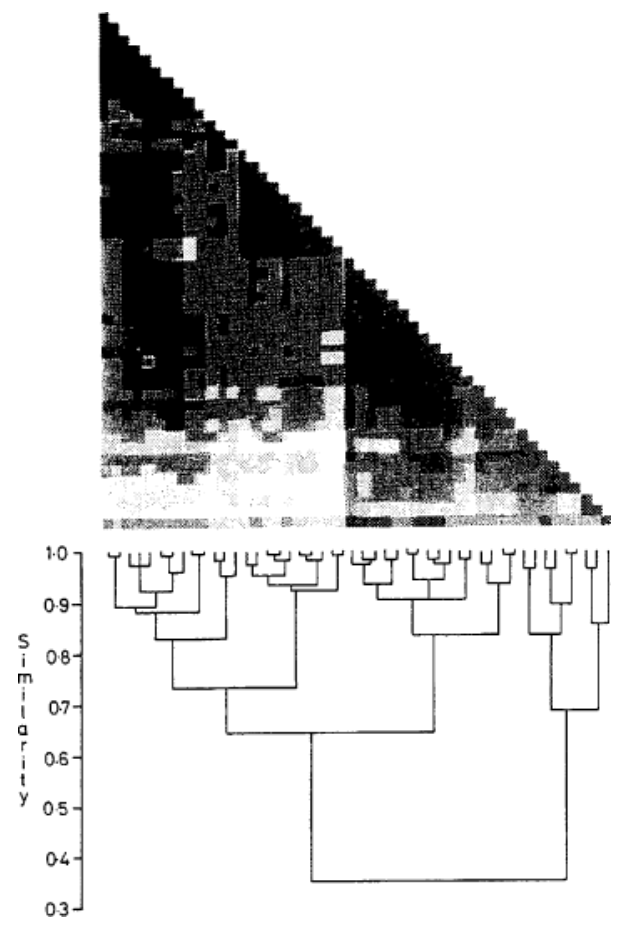

Figure 7. Permuted cluster display from Gower and Digby (1981), following Ling (1973). This display was designed to represent a symmetric similarity/dissimilarity matrix.

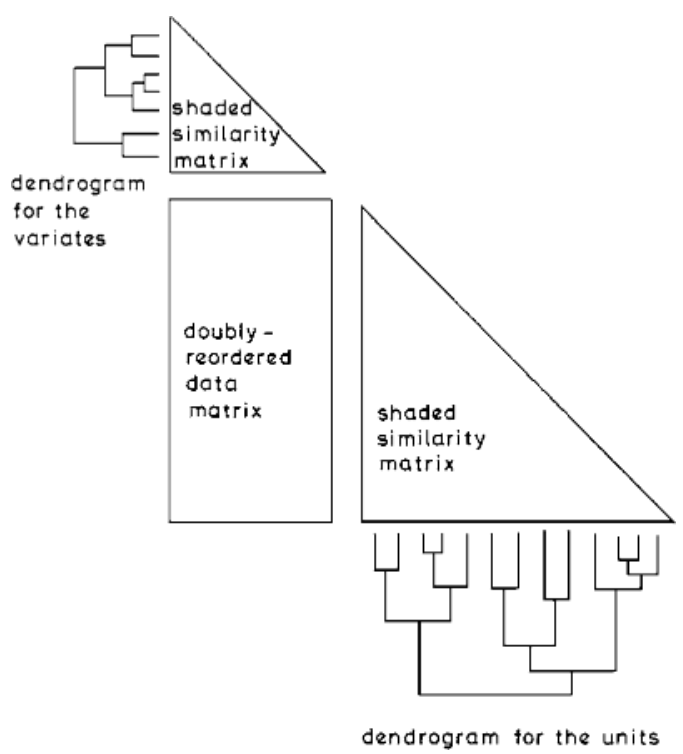

Figure 8. Permuted cluster display framework from Gower and Digby (1981). This is a template for a row/column clustering of a rectangular data matrix. By treating the data as a lower-corner matrix of a square super-matrix, the display reveals both row and column structure.

layout; therefore, we need an additional algorithm to seriate the rows/columns of a clustered matrix. Gruvaeus and Wainer (1972) developed a greedy algorithm that Wilkinson used in the SYSTAT display. Gale, Halperin, and Costanzo (1984) devised an alternative algorithm for this purpose. More recent articles have discussed this problem in detail and specified optimization algorithms with objective functions designed for the task (Wishart 1997; Bar-joseph et al. 2003; Morris, Asnake, and Yen 2003). A desirable aspect of these algorithms is that they yield a total order when it exists (e.g., when the association matrix has Toeplitz form).

\subsection{Appending Trees}

There remains the issue of appending cluster trees to the rectangular data matrix. We have seen examples that append a clustering tree to an association matrix. Gower and Digby (1981) took the next step and appended cluster trees to both row and column association matrices; Figure 8 shows their template. Their layout is in some ways superior to the modern microarray heat map, because it simultaneously displays the row and column similarities/dissimilarities on which the clustering is based. Chen (2002) and others adopted this design.

It is a short step from this design to the layout chosen by the biologists. The first published heat map in this form appeared in Wilkinson (1994). Figure 9 shows a color version of that figure from the SYSTAT manual. By the time the work of Eisen et al. (1998) appeared, tens of thousands of copies of SYSTAT were circulating in the scientific community.

\section{THE FUTURE}

Weinstein (2008) found constructing cluster heat maps a "surprisingly subtle process." His description of these subtleties 


\section{Permuted Data Matrix}

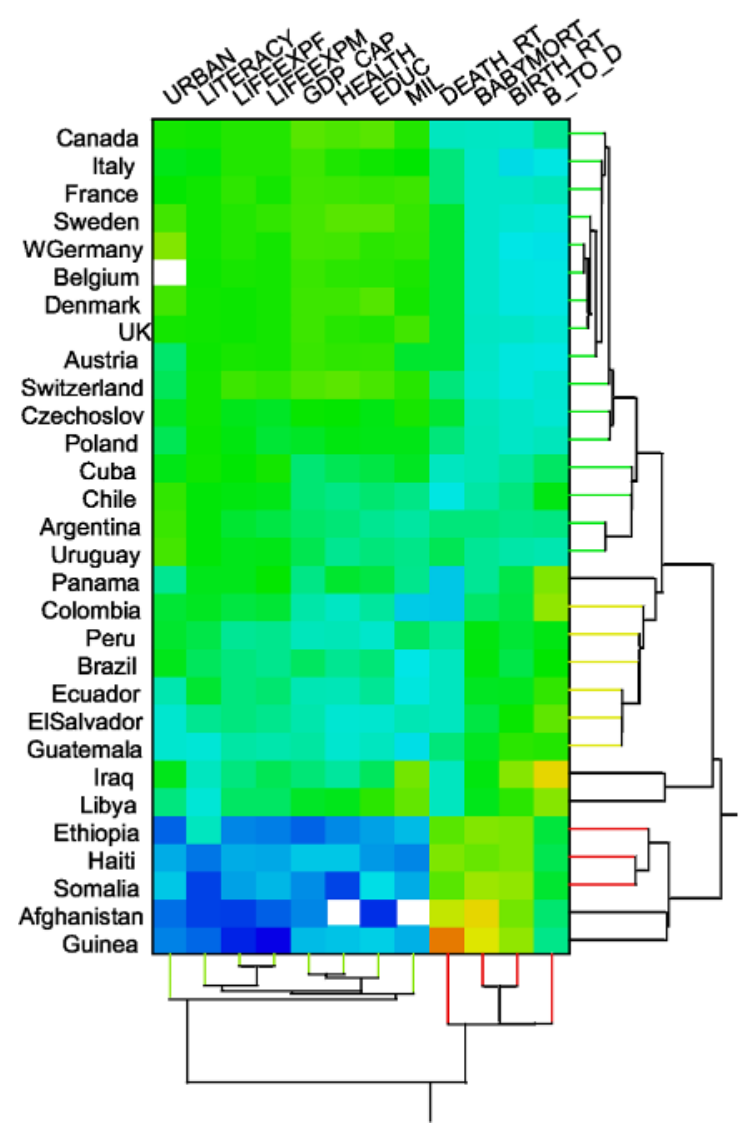

Figure 9. Cluster heat map from Wilkinson (1994). The data are social statistics (i.e., urbanization, literacy, life expectancy for females, GDP, health expenditures, educational expenditures, military expenditures, death rate, infant mortality, birth rate, and ratio of birth to death rate) from a United Nations survey of world countries. The variables were standardized before the hierarchical clustering was performed.

would not surprise a statistician. Those familiar with the cluster literature know that there are issues regarding the choice of a distance measure (e.g., Euclidean, weighted Euclidean, City Block) and the choice of linkage method (e.g., single, complete, average, centroid, Ward). Kettenring (2006) discussed these issues in practice. In addition, Weinstein mentioned the problem of ordering the leaves of the clustering tree, suggesting that "some objective (but, to a degree, arbitrary) rule must be invoked to decide which way each branch will, in fact, swing." As we have mentioned, this is not an arbitrary objective; it is a well-defined seriation problem.

Modern statistical packages implement the heat map display as part of a clustering package (e.g., JMP, SYSTAT), or they make it easy to plot a heat map using any seriation algorithm (e.g., R, Stata). Doing so makes all the options available for clustering or other analytics renderable in a heat map. This flexible architecture underscores the fact that a heat map is a visual reflection of a statistical model, not an arbitrary ordering of row and column cluster trees.

In general, a matrix heat map can be considered a display whose rows and columns have been permuted through an algo- rithm. Many of the recent references cited in this article mention an explicit objective function for evaluating the resulting permutation. A popular seriation loss function is the sum of distances between adjacent rows and columns. We can minimize this function directly on a given data set or use it to evaluate the goodness of a particular heuristic seriation.

Alternatively, we can sample values from known bivariate distributions, randomize rows and columns in the sampled data matrix, and compare the solutions from different seriation algorithms. Wilkinson (2005) generated rectangular matrices whose row and column covariances were determined by five different covariance structures: Toeplitz, Band, Circular, Equicovariance, and Block diagonal. He then randomly permuted rows and columns before applying several different seriation algorithms, including clustering, MDS, and SVD. Overall, SVD recovered the original ordering better than any other method used on all five types of matrices.

These findings suggest that a simple SVD may be the best general seriation method and that cluster methods should be restricted to those data sets for which a cluster model is appropriate. If SVD is chosen, then one should consider recent robust methods for this decomposition (Liu et al. 2003). For microarray data, whether hierarchical clustering-based seriation is more useful than other approaches, despite the popularity of this method, remains an open question.

\section{CONCLUSION}

The cluster heat map did not originate ex nihilo. It came out of a relatively long history of matrix displays, before and after the computer era. As with many graphical methods, the cluster heat map involved a creative synthesis of different graphical representations devised by a number of statisticians.

[Received September 2008. Revised October 2008.]

\section{REFERENCES}

Andrade, M. (2008), “Heatmap,” available at http://en.wikipedia.org/.

Andrich, D. (1978), "A Rating Formulation for Ordered Response Categories," Psychometrika, 43, 357-374.

Bar-joseph, Z., Demaine, E. D., Gifford, D. K., Hamel, A. M., Jaakkola, T. S., and Srebro, N. (2003), "K-Ary Clustering With Optimal Leaf Ordering for Gene Expression Data,” Bioinformatics, 19, 506-520.

Bertin, J. (1967), Sèmiologie Graphique, Paris: Editions Gauthier Villars.

Brinton, W. C. (1914), Graphic Methods for Presenting Facts, New York: The Engineering Magazine Company.

Chen, C. H. (2002), "Generalized Association Plots: Information Visualization via Iteratively Generated Correlation Matrices," Statistica Sinica, 12, 7-29.

Climer, S., and Zhang, W. (2006), "Rearrangement Clustering: Pitfalls, Remedies, and Applications," Journal of Machine Learning Research, 7, 919943.

Czekanowski, J. (1909), “Zur differentialdiagnose der Neandertalgruppe,” Korrespondenzblatt der Deutschen Gesellschaft für Anthropologie, Ethnologie und Urgeschichte, 40, 44-47.

Eisen, M., Spellman, P., Brown, P., and Botstein, D. (1998), “Cluster Analysis and Display of Genome-Wide Expression Patterns," Proceedings of the National Academy of Sciences, 95, 14863-14868.

Friendly, M. (2002), “Corrgrams: Exploratory Displays for Correlation Matrices,” The American Statistician, 56, 316-324. 
Friendly, M., and Kwan, E. (2003), "Effect Ordering for Data Displays," Computational Statistics \& Data Analysis, 43, 509-539.

Gale, N., Halperin, W., and Costanzo, C. (1984), "Unclassed Matrix Shading and Optimal Ordering in Hierarchical Cluster Analysis," Journal of Classification, 1, 75-92.

Goodman, L. (1975), “A New Model for Scaling Response Patterns: An Application of the Quasi-Independence Concept," Journal of the American Statistical Association, 70, 755-768.

Gower, J., and Digby, P. (1981), "Expressing Complex Relationships in Two Dimensions," in Interpreting Multivariate Data, ed. V. Barnett, Chichester, U.K.: Wiley, pp. 83-118.

Gruvaeus, G., and Wainer, H. (1972), "Two Additions to Hierarchical Cluster Analysis," British Journal of Mathematical and Statistical Psychology, 25, 200-206.

Guttman, L. (1950), "The Basis for Scalogram Analysis," in Measurement and Prediction. The American Soldier, Vol. IV, eds. S. A. Stouffer et al., New York: Wiley.

Hage, P., and Harary, F. (1995), "Close-Proximity Analysis: Another Variation on the Minimum-Spanning-Tree Problem," Current Anthropology, 36, 677683.

Hartigan, J. (1974), "BMDP3M: Block Clustering," in BMDP Biomedical Computer Programs, ed. W. Dixon, Berkeley, CA: University of California Press.

\section{(1975), Clustering Algorithms, New York: Wiley.}

Hubert, L. (1974), "Some Applications of Graph Theory and Related NonMetric Techniques to Problems of Approximate Seriation: The Case for Symmetric Proximity Measures," The British Journal of Mathematical and Statistical Psychology, 27, 133-153.

(1976), "Seriation Using Asymmetric Proximity Measures," The British Journal of Mathematical and Statistical Psychology, 29, 32-52.

Kendall, D. (1963), "A Statistical Approach to Flinders Petries Sequence Dating," Bulletin of the International Statistical Institute, 40, 657-680.

Kettenring, J. (2006), “The Practice of Cluster Analysis," Journal of Classification, 23, 3-30.

Lenstra, J. (1974), "Clustering a Data Array and the Traveling Salesman Problem," Operations Research, 22, 413-414.

Liiv, I. (2008), "Pattern Discovery Using Seriation and Matrix Reordering: A Unified View," Ph.D. thesis, Tallinn University of Technology, Department of Informatics, Tallinn, Estonia.
Ling, R. (1973), "A Computer Generated Aid for Cluster Analysis," Communications of the ACM, 16, 355-361.

Liu, L., Hawkins, D., Ghosh, S., and Young, S. (2003), "Robust Singular Value Decomposition Analysis of Microarray Data," Proceedings of the National Academy of Sciences, 100, 13167-13172.

Loua, T. (1873), Atlas statistique de la population de Paris, Paris: J. Dejey.

McCormick, W. T., Schweitzer, P. J., and White, T. W. (1972), "Problem Decomposition and Data Reorganization by a Clustering Technique," Operations Research, 20, 993-1009.

Morris, S. A., Asnake, B., and Yen, G. G. (2003), "Dendrogram Seriation Using Simulated Annealing," Information Visualization, 2, 95-104.

Nie, N. H., Bent, D. H., and Hull, C. H. (1970), SPSS: Statistical Package for the Social Sciences, New York, NY: McGraw-Hill.

Petrie, W. (1899), "Sequences in Prehistoric Remains," The Journal of the Anthropological Institute of Great Britain and Ireland, 29, 295-301.

PNAS (2008), "Most-Cited Articles as of July 1, 2008-Updated Monthly," available at http://www.pnas.org/reports/most-cited.

Robinson, W. (1951), "A Method for Chronologically Ordering Archaeological Deposits," American Antiquity, 16, 293-301.

Rondinelli, D. A. (1980), Spatial Analysis for Regional Development, Tokyo, Japan: The United Nations University.

Siirtola, H., and Mäkinen, E. (2005), "Constructing and Reconstructing the Reorderable Matrix," Information Visualization, 4, 32-48.

Sneath, P. (1957), "The Application of Computers to Taxonomy," Journal of General Microbiology, 17, 201-226.

Weinstein, J. (2008), “A Postgenomic Visual Icon,” Science, 319, 1772-1773.

Wilkinson, L. (1979), “Permuting a Matrix to a Simple Pattern,” in Proceedings of the Statistical Computing Section of the American Statistical Association, Washington, DC: American Statistical Association, pp. 409-412.

(1984), SYSTAT, Version 2, Evanston, IL: SYSTAT Inc.

(1994), SYSTAT for DOS: Advanced Applications, Version 6, Evanston, IL: SYSTAT Inc.

(2005), The Grammar of Graphics (2nd ed.), New York: SpringerVerlag.

Wishart, D. (1997), "ClustanGraphics: Interactive Graphics for Cluster Analysis," Computing Science and Statistics, 29, 48-51. 